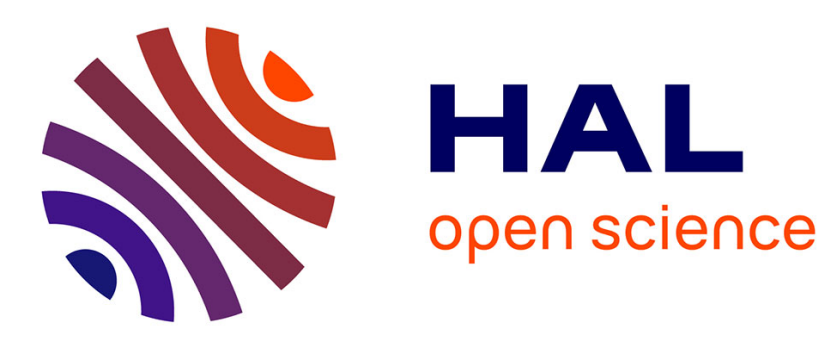

\title{
Diameter constrained overlays with faulty links: equilibrium, stability, and upper bounds
}

Luigi Alfredo Grieco, Mahdi Ben Alaya, Thierry Monteil, Khalil Drira

\section{To cite this version:}

Luigi Alfredo Grieco, Mahdi Ben Alaya, Thierry Monteil, Khalil Drira. Diameter constrained overlays with faulty links: equilibrium, stability, and upper bounds. IEEE Transactions on Circuits and Systems II: Express Briefs, 2016, 63 (8), pp.808-812. 10.1109/TCSII.2016.2530880 . hal-01902728

\section{HAL Id: hal-01902728 \\ https://hal.laas.fr/hal-01902728}

Submitted on 23 Oct 2018

HAL is a multi-disciplinary open access archive for the deposit and dissemination of scientific research documents, whether they are published or not. The documents may come from teaching and research institutions in France or abroad, or from public or private research centers.
L'archive ouverte pluridisciplinaire HAL, est destinée au dépôt et à la diffusion de documents scientifiques de niveau recherche, publiés ou non, émanant des établissements d'enseignement et de recherche français ou étrangers, des laboratoires publics ou privés. 


\title{
Diameter Constrained Overlays with Faulty Links: Equilibrium, Stability, and Upper Bounds
}

\author{
Luigi Alfredo Grieco, Senior Member, IEEE, Mahdi Ben Alaya, Thierry Monteil, and Khalil Drira
}

\begin{abstract}
In network overlays, virtual links among remote processes are usually established to circumvent the limitations of underlying protocols. The resulting dynamics have been recently studied, based on a novel random graph model that assumes no link failures can occur. In that model, the case of faulty links has been only marginally stated to stimulate future research activities. Unfortunately, network overlays are very prone to faulty links, caused by any possible reasons that force a node to loose its connectivity. To bridge this gap, the present brief deepen the implications of faulty links in diameter constrained overlays and demonstrates that: (i) the resulting system has a unique globally stable equilibrium point; (ii) the number of links composing the network is upper bounded in closed form; (iii) the speed of convergence to the equilibrium point is upper bounded in closed form too. These outcomes grant for a stable regime and serve for estimating the overhead incurred by network nodes and sizing them adequately. Finally, to characterize the application bounds of the model, a stochastic analysis of its accuracy has been proposed along with an extensive simulation campaign that encompasses a wide range of scenarios.
\end{abstract}

Index Terms-Graph theory, Topology, Networks, Stability.

\section{INTRODUCTION}

Overlay systems are virtual networks, built upon logical links that are established between remote processes that wish to set up information delivery platforms [1]. They can magnify the capabilities of underlying lower layer protocols and circumvent possible limitations of physical network infrastructures, thus enabling Content Distribution Networks, Peer-to-Peer (P2P) systems, and Machine-to-Machine (M2M) applications [2].

Overlays can be classified as structured and unstructured. The former imposes the position of each node within the topology in order to optimize the data delivery strategy based on the constraints of supported applications. The latter lets nodes progressively discover peers and set up a sub-optimal (yet efficient) mesh topology [3].

Herein, the attention is focused on diameter constrained overlays, supporting delay sensitive applications (e.g., P2P TV [4] and mission critical M2M systems [5]). In these applications, any message received after the expiration of a time deadline is discarded. Therefore, it is important to upper bound the distance (expressed as the number of links composing the path between any couple of nodes) and its maximum value (i.e., the diameter). Hence, in diameter constrained overlays,

L. A. Grieco is with the Department of Electrical and Information Engineering, Politecnico di Bari, Bari, Italy, e-mail: alfredo.grieco@poliba.it

M. B. Alaya and T. Monteil are with CNRS, LAAS, and Univ. de Toulouse, Toulouse, France. K. Drira is with CNRS, LAAS, Toulouse, France. e-mail: \{ben.alaya, monteil, khalil\}@laas.fr the diameter is bounded to a predefined threshold $D$ in order to lower end-to-end communication latencies [1].

The problem of building diameter constrained topologies has been thoroughly afforded in [6] with reference to structured overlays, built upon distributed hash tables (DHT). Recently, the first contribution that describes the dynamics of an unstructured overlay, subject to a constraint on the maximum diameter $D$, has been formulated in [7]. This model is grounded on random graph theory [8]-[13] and assumes ideal conditions (i.e., perfectly reliable and stable links). The case of unreliable links is only marginally addressed in [7] to stimulate future research on the topic. Unfortunately, real overlays are prone to faulty links because any deliberate or unfortunate loss of connectivity would result in a link that disappears from the logical network [2]. To lift this restraint, an enhanced formulation is proposed hereby, that extends the findings in [7] to characterize the equilibrium and dynamics [14] of unstructured overlays with faulty links.

In particular, the approach proposed hereby considers a discrete time process of arrivals, each one associated to a couple of vertices that wish to communicate. Accordingly, a general topology formation mechanism $M$ is formulated, expressing the rules that drive the addition of new edges, obeying to the constraint on the maximum diameter $D$. Also, a probabilistic node fault model is introduced that randomly removes a fraction of links $\lambda$ between any couple of arrivals. Then an approximated state space discrete time model is proposed that describes the evolution of the average network degree subject to $M, D, \lambda$, and the total number of nodes $N$.

The resulting model has been theoretically analyzed to demonstrate that:

- a unique globally stable equilibrium point exists;

- the speed of convergence to the equilibrium point is upper bounded and can be expressed in closed form as a function of $\lambda$ and $N$;

- the amplitude of the trajectory starting from zero links in the network can be upper bounded as a function of $\lambda$ and $N$, too.

These features ensure a stable operating regime of the network and serve for properly estimating the overhead incurred by network nodes and sizing them adequately. Finally, a stochastic analysis of the application bounds of the model has been formulated too along with an extensive simulation campaign that encompasses a wide range of scenarios. The validation clearly shows that: (i) the model is able to predict the system trajectory for small values of $\lambda$; (ii) the upper bound on the number of links is violated in less than $3.2 \%$ of time instants in the worst case. The former result means 
that the model is valid as long as the frequency of link faults is sufficiently smaller than the setup frequency of new data sessions, which is generally satisfied as condition in realistic scenarios. The latter means that, albeit the model is only valid for small values of $\lambda$, the derived upper bounds are robust enough to allow a proper sizing of overlay nodes for any $\lambda$.

The rest of the brief is organized as follows: the model and its properties are presented in Sec. II. Its applicability bounds are theoretically discussed in Sec. III. Then, the model is validated in Sec. IV against computer simulations. The last Sec. V closes the letter and draws future research.

\section{THE MODEL}

Mainly following the notation in [7], the target scenario considered in this brief consists of a graph of $N$ vertices, $n_{q}$ being the $q$-th vertix $(q \in[1, N])$. Furthermore, an ordered sequence of equi-probable ${ }^{1}$ pairs of vertices is considered, among which a path composed of no more than $D$ edges has to be established. The $t$-th couple in the ordered sequence of pairs of vertices is described by $\left(n_{i_{t}}, n_{j_{t}}\right)$. For sake of simplicity, the variable $t$ will be referred to as time from now on. Knowing the $t$-th pair, a new edge is established in the graph if and only if the two vertices $n_{i_{t}}$ and $n_{j_{t}}$ are not reciprocally reachable in less than $D$ edges. To this end, $P_{t-1}$ is defined as the probability that a pair of vertices at time $t$ will not be reciprocally reachable in no more than $D$ edges. Since we are assuming homogeneous conditions, $P_{t-1}$ it is the same for all the possible pairs $\left(n_{i_{t}}, n_{j_{t}}\right)$. In other terms, $P_{t}$ expresses the expected number of links that will be added at time $t+1$. Also, to account for faulty links, the probability $\lambda$ is introduced to express the probability that a link is lost between two time instants (i.e., $\lambda$ is the average fraction of links that will get lost, for any reason, during the establishment of two consecutive data sessions). It is worth to note that, based on these assumptions, at any time instant, any node pair can trigger the creation of a data session with the same probability. As a such, subsequent results will remain valid for any sequence of node pairs.

TABLE I

NOTATION.

\begin{tabular}{l|l} 
Symbol & Meaning \\
\hline \hline$N$ & Number of vertices \\
\hline$k_{t}$ & Average degree at time $t$ \\
\hline$n_{q}$ & $q$-th vertex \\
\hline$D$ & Maximum diameter \\
\hline$\left(n_{i_{t}}, n_{j_{t}}\right)$ & $t$-th pair of vertices wishing to establish a path \\
\hline$l_{t}$ & Number of edges at time $t$ \\
\hline$P_{t-1}$ & $\begin{array}{l}\text { Probability that no path exists shorter than } D+1 \\
\text { edges between the vertices }\left(n_{i_{t}}, n_{j_{t}}\right)\end{array}$ \\
\hline $\operatorname{Pr}\{x\}$ & Probability of event $x$ \\
\hline$\lambda$ & Link Fault Probability \\
\hline \hline
\end{tabular}

Based on these hypotheses, the dynamics of the number of

${ }^{1}$ It is worth to note that equi-probable arrivals and links of the same weight (i.e., homogeneous conditions) are usually assumed in the current literature dealing with diameter constrained graphs [6], [7]. links in the overlay can be expressed as follows:

$$
l_{t+1}=l_{t} \cdot(1-\lambda)+P_{t}
$$

which, considering that the average degree [15] (i.e., the number of edges per vertex) is $k_{t}=\frac{2 \cdot l_{t}}{N}$, can be also expressed as:

$$
k_{t+1}=k_{t} \cdot(1-\lambda)+\frac{2}{N} P_{t}
$$

In [7], it has been demonstrated that, for a sufficiently large $N, P_{t}$ can be expressed as:

$P_{t} \approx \exp \left(-\frac{\left(\frac{2 \cdot l_{t}}{N}\right)^{D+1}-\frac{2 \cdot l_{t}}{N}}{2 \cdot l_{t}-N}\right)=\exp \left(-\frac{1}{N} \cdot \frac{k_{t}^{D+1}-k_{t}}{k_{t}-1}\right)$

so that, the dynamic model (1) or equivalently (2) are completely defined in state space.

To shed some light on the graph dynamics described so far, Figure 1 shows some selected snapshots from the evolution of an overlay with $N=100$ nodes, link fault probability $\lambda=$ 0.01 , and maximum diameter $D=5$.

Theorem 1. For $0<\lambda<1$ and $a$ sufficiently large $N$, the system (2) has a unique globally asymptotically stable equilibrium point

$k_{\infty}$ that satisfies the following inequality ${ }^{2}$ :

$$
k_{\infty} \cdot \lambda=\frac{2}{N} \exp \left(-\frac{1}{N} \cdot \frac{k_{\infty}^{D+1}-k_{\infty}}{k_{\infty}-1}\right)
$$

Proof. The proof of uniqueness has been derived in [7]. To demonstrate the asymptotical stability of the equilibrium point, the definition of asymptotical stability will be used. Accordingly, in $t=t_{0}$ it is applied a perturbation $\Delta_{0}$ to the equilibrium point $k_{\infty}$ and the resulting trajectory is observed. At the next time step, $t_{0}+1$ the state of the system (2) moves to:

$$
k_{\infty}+\Delta_{1}=(1-\lambda) \cdot\left(k_{\infty}+\Delta_{0}\right)+\frac{2}{N} \cdot g\left(k_{\infty}, \Delta_{0}\right)
$$

where $\Delta_{1}$ represents the shift from the equilibrium after one time step from the perturbation and $g\left(k_{\infty}, \Delta_{0}\right)=$ $\exp \left(-\frac{\left(k_{\infty}+\Delta_{0}\right)^{D+1}-\left(k_{\infty}+\Delta_{0}\right)}{N\left(k_{\infty}+\Delta_{0}-1\right)}\right)$.

By replacing (4) in (5), the following expression can be obtained:

$$
\Delta_{1}=(1-\lambda) \cdot \Delta_{0}+\frac{2}{N} \cdot f\left(k_{\infty}, \Delta_{0}\right)
$$

where $f\left(k_{\infty}, \Delta_{0}\right)=\exp \left(-\frac{\left(k_{\infty}+\Delta_{0}\right)^{D+1}-\left(k_{\infty}+\Delta_{0}\right)}{N\left(k_{\infty}+\Delta_{0}-1\right)}\right)-$ $\exp \left(-\frac{k_{\infty}^{D+1}-k_{\infty}}{N\left(k_{\infty}-1\right)}\right)$.

Now, for $\Delta_{0}>0$, it can be noticed that $f\left(k_{\infty}, \Delta_{0}\right)<0$ and hence $\Delta_{1}<(1-\lambda) \cdot \Delta_{0}$. Moreover, $\left|f\left(k_{\infty}, \Delta_{0}\right)\right|<1$, so that, for a sufficiently large $N, \Delta_{1}>0$. Therefore, the following inequalities can be written:

$$
0<\Delta_{1}<(1-\lambda) \cdot \Delta_{0}
$$

${ }^{2}$ The accuracy of the model as a function of $N$ has been investigated in Sec. IV using computer simulations. 


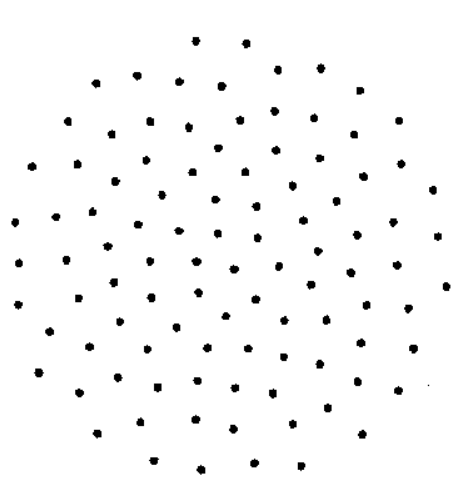

(a)

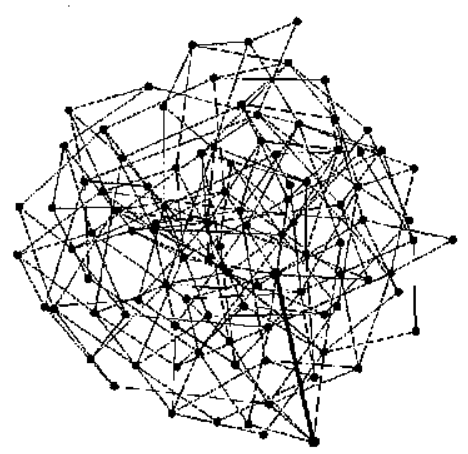

(b)

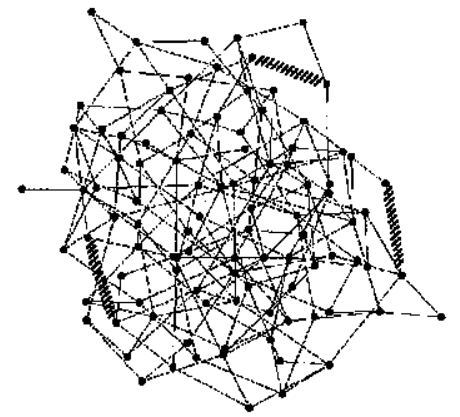

(c)

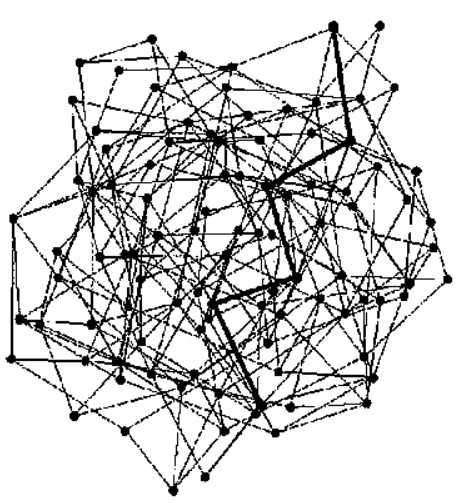

(d)

Fig. 1. Evolution of a random graph $(N=100, \lambda=0.01, D=5)$ : a) initial state; b) a new link is added (bold); c) some links fail (hatched); d) no link is added because a path already exists with less than $D+1$ links (bold).
Conversely, if the perturbation is negative, i.e., $\Delta_{0}<0$, $f\left(k_{\infty}, \Delta_{0}\right)>0$ and hence $\Delta_{1}>(1-\lambda) \cdot \Delta_{0}$. Moreover, since $\left|f\left(k_{\infty}, \Delta_{0}\right)\right|<1$, for a sufficiently large $N$ eq. (6) gives $\Delta_{1}<0$. Summarizing, the following inequalities hold:

$$
(1-\lambda) \cdot \Delta_{0}<\Delta_{1}<0
$$

Now, by putting (7) and (6) together, it yields:

$$
\left|\Delta_{1}\right|<(1-\lambda) \cdot\left|\Delta_{0}\right|
$$

The same approach can be used to derive the next status of the system $k_{0}+\Delta_{2}$, which will result as:

$$
\left|\Delta_{2}\right|<(1-\lambda) \cdot\left|\Delta_{1}\right|<(1-\lambda)^{2} \cdot\left|\Delta_{0}\right|
$$

This result can be extended also to future states, so that the system will converge again to $k_{\infty}$ and, in general, the maximum displacement from the equilibrium is $\Delta_{0}$ during the transient. In other words, for $t \geq t_{0},\left|k_{t}-k_{\infty}\right| \leq$ $\Delta_{0}$, for any perturbation $\Delta_{0}$ applied to the equilibrium state and $k_{t} \rightarrow k_{\infty}$ for $t \rightarrow \infty$.

Theorem 1 tells us that whatever its initial state, the system will naturally converge to one and only one equilibrium point. This condition, which is not straightforward in non linear systems, means that the topology formation mechanism described so far will provide a stable regime with limited perturbations across the unique equilibrium point that solves eq. (4). Furthermore, any perturbation to the equilibrium point applied at the time instant $t_{0}$ will be damped quicker than the function $(1-\lambda)^{t-t_{0}}$. This latter information is also useful to predict, at steady state, the dynamics of possible perturbations across the equilibrium and help sizing the overlay.

Besides the steady state behavior, it is also important to study the natural trajectory of the overlay (i.e., the evolution of the overlay starting from an initial state of zero links).

Theorem 2. For any $N$, the trajectory of system (2) having as initial point $k_{0}=0$ is upper bounded as follows:

$$
k_{t} \leq \frac{2}{N} \cdot \frac{1-(1-\lambda)^{t}}{\lambda}<\frac{2}{N \cdot \lambda}
$$

Proof. For $k_{0}=0, k_{1}$ can be derived from (2) thus obtaining $k_{1}=\frac{2}{N}$. Following the same rationale, $k_{2}=\frac{2}{N} \cdot[1-\lambda+$ $\left.P_{1}\right]$ and $k_{3}=\frac{2}{N} \cdot\left[(1-\lambda)^{2}+(1-\lambda) P_{1}+P_{2}\right]$. In general, $k_{t}=\frac{2}{N} \sum_{l=0}^{t-1}(1-\lambda)^{l} \cdot P_{t-l-1} \leq \frac{2}{N} \sum_{l=0}^{t-1}(1-\lambda)^{l}$, since $P_{l} \leq 1 \forall l$

Corollary 1. The equilibrium point of system (2) is upper bounded as follows:

$$
k_{\infty} \leq \frac{2}{\lambda \cdot N}
$$

Proof. The proof can be easily derived from Theorem 1 with $t \rightarrow \infty$.

Remark 1. Considering Corollary 1 and that $k=\frac{2 \cdot l}{N}$, the number of links $\left(l_{\infty}\right)$ at steady state is no larger than $1 / \lambda$.

Remark 2. Considering Theorem 2 and that $k=\frac{2 \cdot l}{N}$, the number of links $\left(l_{t}\right)$ is bounded by $1 / \lambda$ for the trajectory starting with zero links at time zero. 
Remark 3. Considering Theorem 2, the speed of convergence to the equilibrium point is driven by $(1-\lambda)^{t}$.

Theorem 2 and the subsequent remarks and corollaries tell us that it is possible to upper bound the number of links in the overlay (and hence the equilibrium point) for the natural trajectory in closed form. This property is very relevant because knowing the single parameter $\lambda$, it is possible to forecast an upper limit of the number of links, whatever $N$ and $D$. Also in this case, this upper bound is useful to predict the average degree of the overlay (i.e., the average number of links per vertex) and size nodes adequately. Moreover, Theorem 2 confirms the findings of Theorem 1 that refer to the speed of convergence, that is driven by the function $(1-\lambda)^{t}$. Moreover, with reference to Remark 2, it is worth to note that the number of links cannot grow faster than one link per time slot, whatever $N$, so that also its upper bound is independent from $N$

In any case, it is worth to note that the results of both Theorems are approximated because the recursive model (1) is based on the assumption that the expected number of links at the next time step, knowing the number of links at the current step, it is an accurate approximation of the actual number of links at the next time step. In addition, even if this one step approximation is accurate, i.e., it is characterized by a small error, the recursion in eq. (1) could magnify the overall estimation error. For this reason, in the next Section, the accuracy of the model will be studied through a stochastic analysis of the standard deviation and the coefficient of variation of the key variables of the system.

\section{MODEL ACCURACY}

As for any model, it is important to evaluate the applicability of Eqs. (2) and (1) to the study of a real overlay. To this end, the conditional random variable $l_{t+1} \mid l_{t}$ (i.e., $l_{t+1}$ assuming that $l_{t}$ is known) is introduced hereby as follows:

$$
l_{t+1} \mid l_{t}=l_{t}+A_{t}-F_{t}
$$

where $A_{t}$ and $F_{t}$ are independent random variables that model the number of links added and the number of links fallen during the last time step, respectively. In particular, $A_{t}$ is equal to one with probability $P_{t}$ and to zero with probability $1-P_{t}$. On the other hand, $F_{t}$ can assume any value $f$ in the range $\left[0, l_{t}\right]$, with probability $C_{l_{t}, f} \lambda^{f} \cdot(1-\lambda)^{l_{t}-f}$, with $C_{l_{t}, f}=$ $\frac{l_{t} !}{f ! \cdot\left(l_{t}-f\right) !}$.

Now, the expected value of $l_{t+1} \mid l_{t}$ can be evaluated as:

$$
\mu\left(l_{t+1} \mid l_{t}\right)=l_{t}+P_{t}-l_{t} \cdot \lambda .
$$

It is worth noticing that $\mathrm{Eq}$. (1) is equal to the average value of the conditional random variable $l_{t+1} \mid l_{t}$, so that the value of $l_{t+1}$ provided by Eq. (1) is an unbiased one step estimate of the actual value $l_{t+1}$.

To understand the accuracy of such an estimate, it is necessary to evaluate the variance of $l_{t+1} \mid l_{t}: \sigma^{2}\left(l_{t+1} \mid l_{t}\right)$. Since, $A_{t}$ and $F_{t}$ are independent of each other, $\sigma^{2}\left(l_{t+1} \mid l_{t}\right)$ can be computed as:

$$
\sigma^{2}\left(l_{t+1} \mid l_{t}\right)=\sigma_{A_{t}}^{2}+\sigma_{F_{t}}^{2}
$$

Now, since $A_{t}$ and $F_{t}$ are binomial random variables, it yields $\sigma_{A_{t}}^{2}=P_{t} \cdot\left(1-P_{t}\right)$ and $\sigma_{F_{t}}^{2}=l_{t} \cdot \lambda \cdot(1-\lambda)$. As a consequence, the coefficient of variation $C o V=\frac{\sigma\left(l_{t+1} \mid l_{t}\right)}{l_{t}(1-\lambda)+P_{t}}$ is equal to:

$$
C o V=\frac{\sqrt{P_{t} \cdot\left(1-P_{t}\right)+l_{t} \cdot \lambda \cdot(1-\lambda)}}{l_{t} \cdot(1-\lambda)+P_{t}}
$$

The coefficient $\mathrm{CoV}$ measures the degree of dispersion around the expected value $\mu$, so that it is highly desirable it is much less than one. Now, the $\mathrm{CoV}$ will be analyzed in different cases. In this analysis, it will be assumed that $\lambda \leq 0.5$, which means that a link is more likely to remain active than falling in each time step. This assumption is motivated also by Theorem 2 , which would result in less than two active links at steady state for $\lambda>0.5$. By deriving Eq. (16) with respect to $\lambda$, it is immediate to discover that the maximum value of the $\mathrm{CoV}$ is obtained for $\lambda=0.5$, and it is:

$$
\operatorname{CoV}_{M}=\frac{\sqrt{P_{t} \cdot\left(1-P_{t}\right)+\frac{l_{t}}{4}}}{\frac{l_{t}}{2}+P_{t}}
$$

It is worth to note that $C o V_{M}$ is upper bounded by $\sqrt{\frac{1}{l_{t}^{2}}+\frac{1}{l_{t}}}$. This means that the one step predictor used to derive the overlay dynamics is very accurate for sufficiently large values of $l_{t}$. Now, considering that from Theorem $2 l_{t} \leq \frac{1}{\lambda}$, it results that increasing $\lambda$ will worsen the accuracy of the model. For this reason, the proposed model can be considered valid when $\lambda<<1$.

Notice that, this assumption does not limit the applicability of the model because the hypothesis $\lambda<<1$ simply means that the average frequency at which links fall is much smaller than the average rate at which new data sessions are established, which is quite common in practice. Moreover, in the next section it will be shown that while the model is accurate for $\lambda<<1$, the bound $1 / \lambda$ on the number of links, derived in Theorem 2 and Remark 2 , remains valid for almost all values of $\lambda$ in the considered scenarios.

\section{VALIDATION}

To validate the model (1) or equivalently (2), an ad hoc simulator has been developed in Matlab, similarly to what is done in [7]. The analysis carried out in the previous section showed that the accuracy of the one step prediction provided by (1) increases by decreasing $\lambda$. Herein, the accuracy of the model is evaluated over multiple time steps. In particular, an overlay composed by $N$ nodes (with $N \in[64,1024]$ ) with $D \in[3,6]$ and $\lambda \in\left[10^{-6}, 10^{-1}\right]$ has been simulated. At the end of each simulation, the dynamics of the number of links resulting from the simulator and from Eq. (1) have been compared and the average absolute relative error is computed. Table II reports the minimum and maximum values of the average absolute relative error obtained by varying $D$ for any given couple of values $(N, \lambda)$ (selected results are also shown in Fig. 2). This analysis clearly shows that, as expected from the findings of the previous section, the model is valid for values of $\lambda$ much less than one. 
TABLE II

VALIDATION RESULTS: AVERAGE ABSOLUTE RELATIVE ERROR (\%)

\begin{tabular}{l|l|l|l|l|l|l}
$N$ & $\lambda$ & $\lambda$ & $\lambda$ & $\lambda$ \\
$10^{-6}$ & $10^{-5}$ & $10^{-4}$ & $\begin{array}{l}\lambda \\
10^{-3}\end{array}$ & $\begin{array}{l}\lambda \\
10^{-2}\end{array}$ & $\begin{array}{l}\lambda \\
10^{-1}\end{array}$ \\
\hline \hline 64 & {$[\overline{3.4,4.8}$} & $2.7,4.6$ & {$[2.4,6.7$} & {$[2.4,12.8$} & {$[6.5,8.7]$} & $>100$ \\
\hline 128 & {$[1.5,5.5]$} & {$[1.2,5.9]$} & $2.3,10]$ & {$[2.2,13.6]$} & {$[16,30]$} & $>100$ \\
\hline 256 & {$[1.3,4.1]$} & {$[1.8,8.2]$} & $1,12.5]$ & {$[2.8,9.5]$} & $>100$ & $>100$ \\
\hline 512 & $1.2,5.5]$ & {$[1.6,10]$} & $0.9,13.4$ & {$[3.1,7.9]$} & $>100$ & $>100$ \\
\hline 1024 & {$[1.5,7.4]$} & {$[0.4,12.4$} & $0.7,11.3$ & {$[14.2,24.6$} & $>100$ & $>100$ \\
\hline \hline
\end{tabular}

TABLE III

Validation Results: MaXimum PERCENTAge of Times the bound $1 / \lambda$ IS VIOLATED.

\begin{tabular}{|c|c|c|c|c|c|c|}
\hline$N$ & $\begin{array}{l}\lambda \\
10^{-6}\end{array}=$ & $\begin{array}{l}\lambda \\
10^{-5}\end{array}=$ & $\begin{array}{l}\lambda \\
10^{-4}\end{array}=$ & $\begin{array}{l}\lambda \\
10^{-3}\end{array}=$ & $\begin{array}{l}\lambda \\
10^{-2}\end{array}=$ & $\begin{array}{l}\lambda \\
10^{-1}\end{array}=$ \\
\hline$\overline{664}$ & $\overline{0} 0$ & $\overline{0}$ & $\overline{0}$ & $\overline{0}$ & $\overline{0}$ & 3.2 \\
\hline 128 & 0 & 0 & 0 & 0 & 0.33 & 1.76 \\
\hline 256 & 0 & 0 & 0 & 0 & 1.57 & 0.76 \\
\hline 512 & 0 & 0 & 0 & 0 & 0.63 & 0.53 \\
\hline 1024 & 0 & 0 & 0 & 1.17 & 0.36 & 0.46 \\
\hline
\end{tabular}

In particular, the absolute relative error is below $15 \%$ for $\lambda \leq 10^{-4}$, which makes perceive the proposed model a very powerful tool to characterize unstructured overlays with faulty links. Notice that $\lambda=10^{-4}$ means that the link failure frequency is four orders of magnitude less than the set up rate of new data sessions. In other words, assuming $\lambda<<1$ does not limit the applicability of the model since, usually, link failures are very sporadic if compared to the rate at which data sessions are established.

Finally, to provide a further insight, the percentage of time instants in which the bound $1 / \lambda$ of Theorem 2 is violated is registered too (see Table III). With reference to this last investigation, it is worth to remark that this percentage is lower than $3.2 \%$, so that also in those scenarios in which the absolute relative error is pretty high (i.e., $\lambda \geq 10^{-3}$ ), the bound $1 / \lambda$ on the number of links can be considered valid and useful to size the overlay for any $\lambda$.

\section{Conclusion}

A novel model has been proposed to characterize unstructured overlays for delay sensitive applications. The application bounds of the model have been discussed using theoretical arguments and computer simulations. Remarkably, it has been shown that this kind of overlays converge to a unique globally stable equilibrium point having less than $1 / \lambda$ links, with $\lambda$ being the probability to loose a link during the establishment of two consecutive data session. Moreover, it has been demonstrated that the speed of convergence to the equilibrium point only depends on $(1-\lambda)$. Knowing these bounds it becomes possible to size computing resources, e.g., memory and CPU, in overlay nodes in order to accomodate the overhead incurred by networking primitives. Future research will explore heterogeneous conditions to broaden the scope of the model.

\section{REFERENCES}

[1] J. Kurose and K. Ross, Computer Networking: A Top-Down Approach. Pearson Education, Limited, 2012, 6th edition.

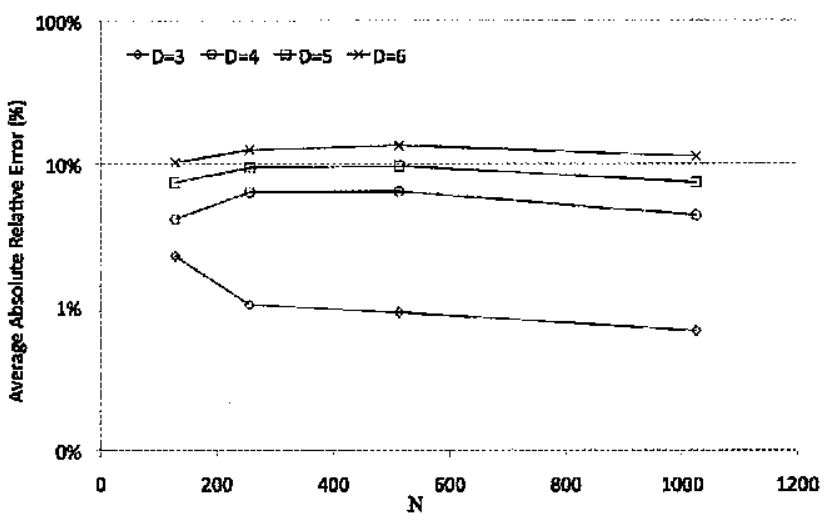

(a)

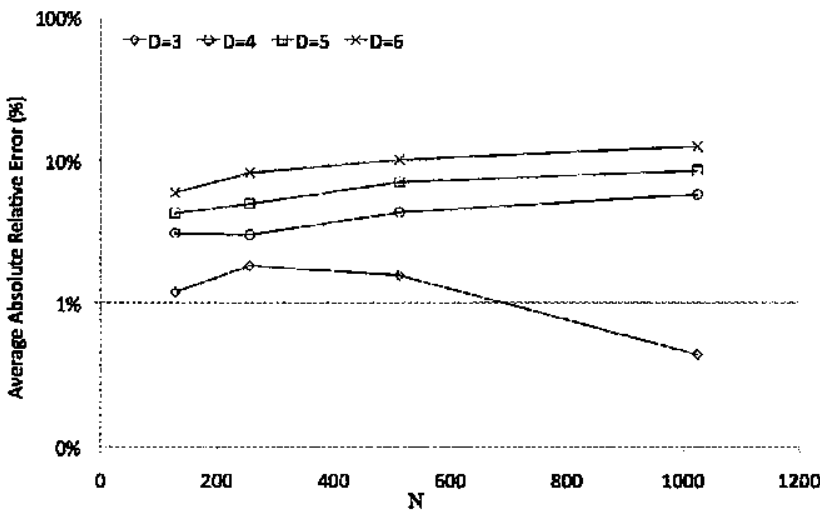

(b)

Fig. 2. Average absolute relative errors of the model (1) for (a) $\lambda=10^{-4}$ and (b) $\lambda=10^{-5}$.

[2] L. Massoulie, A.-M. Kermarrec, and A. J. Ganesh, "Network awareness and failure resilience in self-organising overlay networks," in 22nd IEEE Int. Symposium on Reliable Distributed Systems, 2003, pp. 47-55.

[3] E. K. Lua, J. Crowcroft, M. Pias, R. Sharma, and S. Lim, "A survey and comparison of peer-to-peer overlay network schemes" Communications Surveys Tutorials, IEEE, vol. 7, no. 2, pp. 72--93, Second 2005.

[4] P. Veglia and D. Rossi, "Performance evaluation of P2P-TV diffusion algorithms under realistic settings," Peer-to-Peer Networking and Application, pp. 26-45, 2013.

[5] L. A. Grieco, M. B. Alaya, T. Monteil, and K. Drira, "Architecting information centric ETSI-M2M systems," in Proc. of IEEE PerCom, 2014, pp. 211-214.

[6] D. Loguinov, J. Casas, and X. Wang, "Graph-theoretic analysis of structured peer-to-peer systems: Routing distances and fault resilience," IEEE Transactions on Networking, vol. 13, no. 5, pp. 1107-1120, 2005.

[7] L. A. Grieco, M. B. Alaya, T. Monteil, and K. Drira, "A dynamic random graph model for diameter constrained topologies in networked systems," IEEE Transactions on Circuits and Systems II, vol. 61, no. 12, pp. 982986, 2014.

[8] P. Erdos and A. Renyi, "On random graphs," I. Publ. Math. Debrecen, vol. 6, pp. 290-297, 1959.

[9] S. Boccaletti, V. Latora, Y. Moreno, M. Chavez, and D.-U. Hwanga, "Complex networks: Structure and dynamics," PHYSICS REPORTS, vol. 424 , no. 4-5, pp. 175-308, 2006.

[10] M. Babaei, H. Ghassemieh, and M. Jalili, "Cascading failure tolerance of modular small-world networks" IEEE Transactions on Circuits and Systems II: Express Briefs, vol. 58, no. 8, pp. 527-531, Aug 2011.

[11] Y. Chen, W. Yu, F. Li, and S. Feng, "Synchronization of complex networks with impulsive control and disconnected topology" IEEE Transactions on Circuits and Systems II: Express Briefs, vol. 60, no. 5, pp. 292-296, May 2013.

[12] T. Bonald, L. Massoulie, F. Mathieu, D. Perino, and A. Twigg, "Epi- 
demic live streaming: Optimal performance trade-offs," in SIGMETRICS. ACM, 2008, pp. 325-336.

[13] R. J. Lobb, A. P. C. da Silva, E. Leonardi, M. Mellia, and M. Meo, "Adaptive overlay topology for mesh-based P2P-TV systems," in NOSSDAV. ACM, 2009, pp. 31-36.

[14] K. J. Astrom and B. Wittenmark, Computer controlled systems: theory and design, 3rd ed. Prentice Hall, 1995.

[15] P. Erdos and A. Renyi, "On the evolution of random graphs," Mat Kutato Int. Kozl, vol. 5, no. 17, pp. 17-60, 1960. 